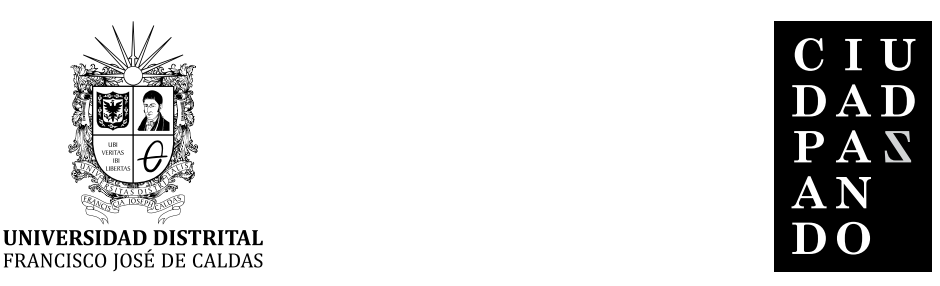

C I U

DA D

PA S

$\mathbf{A} \mathbf{N}$

FRANCISCO JOSÉ DE CALDAS

DOSSIER

Artículo de investigación científica

\title{
Contra la indiferencia: un llamado para la participación civil en el posconflicto en Colombia $^{1}$
}

Against indifference: a call for civil engagement in postconflict in Colombia

Contra a indiferença: um chamado para o engajamento civil no post-conflito na Colômbia

\section{Giuseppe Feola ${ }^{2}$}

Para citar: Feola, G. (2018). Contra la indiferencia: un llamado para la participación civil en el posconflicto en Colombia. Revista Ciudad Paz-ando, 11(1), pp. 51-61. doi: https://doi.org/10.14483/2422278X.12622

Fecha de recepción: 23 de octubre de 2017

Fecha de aprobación: 13 de marzo de 2018

1 Algunas de las ideas presentadas en este artículo surgieron de conversaciones con Jaime Suzunaga, Jenny Soler, Jaime Díaz, Nelson Cabrera, Olga Hernández y Alejandra Osejo. Agradezco a Mike K. Goodman y Jaime Díaz por su apoyo y comentarios constructivos sobre una versión anterior de este artículo. Mi agradecimiento a todos los sogamoseños y sogamoseñas que han intercambiado sus opiniones sobre el proceso de paz conmigo, y a la Real Sociedad Geográfica con IBG para financiar el trabajo de campo (Environment and Sustainability Research Grant 01/17).

2 Doctor del Departamento de Geografía, Universidad de Zurich, Suiza. Profesor asistente del Instituto Copernicus de Desarrollo Sostenible, Universidad de Utrecht, Paises Bajos. Afiliado del Departamento de Geografia y Ciencias Ambientales de la Universidad de Reading, Reino Unido. Correo electrónico: g.feola@uu.nl 


\section{RESUMEN}

El acuerdo de paz del 2016 fue recibido con indiferencia por gran parte de la población colombiana, con ello en mente, el presente texto sugiere que la indiferencia tiene que ver con fracturas estructurales en la sociedad. Se presentan cuatro tesis contra la indiferencia: (a) las limitaciones del acuerdo de paz no eliminan su potencial de generar cambio; (b) la presión de la sociedad civil puede responsabilizar a los que están en poder por la realización del acuerdo de paz; (c) la sociedad civil puede apropiarse del proceso de paz e influir en su construcción; (d) la construcción de la paz es una oportunidad para dar forma a un país más diverso, inclusivo, democrático, digno y ambientalmente consciente.

Palabras clave: Colombia, construcción de paz, posconflicto sociedad civil.

\section{ABSTRACT}

The peace agreement of 2016 was received with substantial indifference by a large part of the Colombian population. This article suggests that the aforementioned indifference is related to rooted fractures in the Colombian Society. Four thesis against indifference are put as follows: (i) the limitations of the peace agreement do not alter its potential for change; (ii) pressure from the civil society and the public can hold those in power accountable for the realisation of the peace agreement; (iii) the civil society and the public can appropriate the peace process and have a positive incidence in the peacebuilding; (iv) peacebuilding is the opportunity of not only to build peace, but also to newly shape a more diverse, inclusive, democratic, dignifying and environmentally-conscious Colombia.

Keywords: civil society, Colombia, peacebuilding, post-conflict.
O acordo de paz de foi recebido com indiferença por grande parte da população colombiana. Este artigo sugere que a indiferença tem a ver com fraturas estruturais na sociedade colombiana. Quatro teses são apresentadas contra a indiferença: (i) as limitações do acordo de paz não eliminam seu potencial para gerar mudanças; (ii) a pressão da sociedade civil e do público pode responsabilizar os responsáveis pelo cumprimento do acordo de paz; (iii) a sociedade civil e o público podem se apropriar do processo de paz e influenciar a construção da paz; (iv) a construção da paz é uma oportunidade não só para construir a paz, mas também para moldar um país mais diversificado, inclusivo, democrático, digno e ambientalmente consciente.

Palavras-chave: Colômbia, construção da paz, pós-conflito, sociedade civil. 


\section{La anomalía de la indiferencia}

\author{
Acaso el final de esta Guerra sea por fin el comienzo de \\ ese país nuevo que tanto hemos esperado. \\ William Ospina, Pa que se acabe esta vaina
}

El acuerdo de paz firmado en 2016 "para poner fin al conflicto y construir una paz estable y duradera" (Alto Comisionado para la Paz, 2016), por el Gobierno colombiano y el grupo insurgente Fuerzas Armadas Revolucionarias de Colombia Ejército del Pueblo (Farc-Ep), ha sido muy favorablemente recibido en un ámbito internacional. El presidente colombiano, Juan Manuel Santos, fue galardonado con el Premio Nobel de la Paz 2016 por sus esfuerzos decididos por poner fin a la guerra civil de más de 50 años; otras entidades internacionales como las Naciones Unidas, a través de su verification mission, y la Unión Europea, a través de su trust fund, se han comprometido a apoyar la aplicación del acuerdo y desempeñan un papel activo en la supervisión, financiación y apoyo al esfuerzo de construcción de paz. Por otro lado, las innovadoras soluciones legales, políticas y prácticas que llevaron al acuerdo de paz son lecciones que dan una luz de esperanza a otros países plagados de guerras prolongadas (Herbolzheimer, 2016; Siegfried, 2016).

Entre los colombianos, sin embargo, el acuerdo de paz se recibió con sentimientos más contradictorios. La población se ha polarizado, como lo demuestran claramente los resultados del plebiscito que ocurrió en octubre de 2016: los votos en contra del acuerdo - 50,21\% - superaron ligeramente a los a favor $-49,78 \%-$ (El Pais, $2016)^{3}$. Como señalaron muchos comentaristas, esta polarización tenía una importante dimensión geográfica; en general, las regiones más afectadas por el conflicto armado votaron a favor del acuerdo, mientras que los centros urbanos y las regiones menos afectadas votaron en su mayoría contra del acuerdo firmado por el Gobierno colombiano y las Farc-Ep. Además, dada la naturaleza histórica del acuerdo y, por consiguiente, su impacto potencial sobre el país, un aspecto que fue particularmente sorprendente para muchos observadores exteriores fue la baja participación: el $62,52 \%$ de los colombianos elegibles optaron por no ejercer este derecho fundamental. Al menos el 51\% de los residentes del Departamento de Casanare decidieron no votar en esta ocasión, junto con más del $80 \%$ de los que viven en La Guajira y figuras similares entre los colombianos que viven en el exterior.

Unos comentaristas han sugerido que la propagación de la desinformación, y una deficiente pedagogía de la paz, pueden haber contribuido a la baja participación en el plebiscito. Algunas personas desconfiaron del propio proceso de negociación que condujo a la firma del

\footnotetext{
3 Un acuerdo de paz revisado fue posteriormente aprobado por votación en el Congreso (The Guardian, 2016, 01 de diciembre, en línea)
}

acuerdo, el cual no encontraron transparente o satisfactorio por varias razones, entre las cuales cuentan las nociones de justicia transicional (Cárdenas Ruiz, 2013; Nasi y Rettberg, 2016). También se ha argumentado que existen definiciones diferentes y parcialmente incompatibles de la paz, las cuales se reflejan en objetivos políticos divergentes (Rettberg, 2003); sin embargo, la mayoría de los factores mencionados parecen explicar más apropiadamente la marcada división entre las personas a favor o en contra del acuerdo de paz que el nivel de desinterés en tal proceso histórico en la historia colombiana y mundial.

¿Puede el nivel de desinterés ser atribuido únicamente a un descontento generalizado con la política (lo que no es raro hoy en día en otros países de América Latina y en otras partes del mundo), tal vez agravado por altos niveles de corrupción que excluyen a la participación en el proceso democrático, como sugieren otros comentaristas (Diaz, 2017)? ¿O acaso los colombianos fueron víctimas de la política de la 'posverdad' (González, 2017) y, por lo tanto, simplemente no pudieron apreciar las apuestas de esta votación? ¿Fue tan alto nivel de desinterés un indicador de indiferencia, y si fue así por qué tantas personas fueron indiferentes al acuerdo de paz? ¿Cuáles fueron las raíces sociales, culturales, e históricas de esta indiferencia? ¿Tal desinterés socavará el esfuerzo de consolidación de la paz? Y, si la indiferencia es una amenaza para un cambio potencialmente histórico, ¿cuáles son algunas razones por las cuales los colombianos deberían participar activamente en el esfuerzo de construcción de paz?

Este artículo representa un esfuerzo para responder estas preguntas. Lo cual se hizo a través de la reflexión sobre varias conversaciones recientes con activistas sociales y medioambientales, funcionarios públicos, periodistas, empresarios y líderes sociales, agricultores y ciudadanos comunes con se tuvo el privilegio de hablar mientras se desarrollaba un proyecto 4 sobre la agricultura periurbana en Sogamoso, Boyacá. Durante aquellas conversaciones, sogamoseños y sogamoseñas han puesto al descubierto una serie de cuestiones que no solo proporcionan una aclaración de la baja participación en el plebiscito de octubre de 2016, sino que también arrojan luz sobre los desafíos que se avecinan en el esfuerzo de construcción de la paz. El objetivo de este artículo es ofrecer al mismo tiempo una explicación de por qué tantas personas han sido indiferentes a este histórico acuerdo de paz, una reflexión sobre lo que significa esta indiferencia para la perspectiva de la consolidación de la paz y algunos argumentos para contrarrestar esa indiferencia. Sin embargo, es claro que este artículo no habla solo de Sogamoso o Boyacá, sino de otras regiones y territorios que no han

\footnotetext{
4 El proyecto, financiado por la Royal Geographical Society (Reino Unido), es una colaboración entre la Universidad de Reading y la Fundación Jischana Huitaca. El sitio web del proyecto es: https://blogs.reading.ac.uk/governing-sustainable-agri-food-systems-in-colombia/
} 
sido epicentros del conflicto armado en Colombia. Se espera que las ideas propuestas aquí puedan ayudar a romper la indiferencia compartida por tantos colombianos y moverlos a formar parte de un futuro pacífico para su país, además de darle forma.

\section{Explicando la indiferencia}

¿Son tantos los colombianos realmente indiferentes al acuerdo de paz? En realidad, cuando se habla de la paz como un valor o principio en términos generales, casi ninguna persona expresó oposición. Como muchas personas lo afirmaron en términos bastante generales: "la paz es mejor que la guerra". Sin embargo, al pensar en esta paz que sigue a este conflicto en particular, la mayoría de las personas sintieron que el acuerdo de paz y el proceso de construcción de paz no afecta mucho su organización, su comunidad o a ellos mismos como individuos. La gran mayoría de las personas no habían leído el acuerdo de paz, ni tampoco ninguno de los materiales de información simplificados (y útilmente más cortos) como aquellos preparados por el Alto Comisionado para la Paz.

Más que eso, la actitud general hacia el acuerdo de paz, el proceso de construcción de paz y su impacto potencial en Sogamoso es abiertamente de escepticismo, quizás de resignación y, esencialmente, de indiferencia. Esto se desvía de manera impresionante con las opiniones mucho más optimistas (aunque a menudo críticas) que han dominado gran parte de los informes internacionales sobre el proceso de paz en Colombia (The Guardian, 2016). El acuerdo de pazy el proceso de construcción de paz actualmente en desarrollo parece estar lejos de la experiencia cotidiana de la gran mayoría de las personas con las que se habló. ¿Por qué? ¿Por qué tantas personas, ciudadanos y ciudadanas comunes, así como líderes empresariales y sociales en Sogamoso han venido manteniendo esta opinión del proceso de paz? Las razones pueden agruparse bajo dos razonamientos aparentemente opuestos: "nunca ha habido conflicto en Sogamoso" y "nunca habrá paz en Colombia".

\section{Nunca ha habido conflicto en Sogamoso}

Es indudable que Sogamoso, como la mayor parte del departamento de Boyacá, ha estado entre los menos afectados por cualquier forma de violencia durante el conflicto armado en el país (CNHM, 2015; Rodríguez, 2009; Salas, 2015). Las personas se refirieron explícita o implícitamente a este hecho cuando minimizaron la presencia de un conflicto armado en Sogamoso y sus alrededores. El conflicto se considera como un fenómeno que interesó más a las zonas rurales que a las urbanas, y que afectó a Boyacá mucho menos que a otras regiones, como el Llano Oriental. Por otra parte, la impresión general es que las iniciativas de consolidación de la paz (por ejemplo, en los sectores de la vivienda o agrícola), en su mayoría, se dirigen a las zonas directamente afectadas por la guerra. lo anterior desincentiva el compromiso con el acuerdo de paz y el proceso de construcción de paz en Sogamoso. En suma, la mayoría de los sogamoseños y sogamoseñas no habían visto y no esperaban ver ningún cambio local sustancial como resultado del fin del conflicto armado o del corriente proceso hacia la paz.

\section{Nunca habrá paz en Colombia}

La indiferencia hacia el acuerdo de paz también se justifica magnificando las limitaciones y las posibles dificultades para realizar el paso hacia una paz estable y duradera. El Gobierno colombiano se ha desempeñado tradicionalmente mejor al legislar políticas - tanto ambientales como sociales o económicas-que a hacerlas cumplir; generalmente, la presencia del Estado colombiano en zonas de conflicto y en zonas rurales ha sido históricamente débil (Feola, 2017), esto ha contribuido a perpetuar y ampliar la brecha urbano-rural (Jaramillo, 2006; Rodríguez et al., 2016). Por otra parte, el acuerdo de paz es multifacético, incluso en sus etapas iniciales el proceso de construcción de paz ya se enfrenta a problemas que pueden llegar a ser barreras fatales. Por ejemplo, el acuerdo de paz solo involucra a las Farc-Ep, pero no involucra a otros grupos todavía activos, como el Ejército de Liberación Nacional (ELN).

Mientras se están realizando conversaciones iniciales entre el Gobierno colombiano y el ELN sobre el inicio de un proceso de negociación análogo al que dio lugar al acuerdo de paz firmado por el Gobierno y las Farc-Ep, al momento de redactar este artículo, el resultado de esta negociación es incierta; de hecho, algunos observadores temen que el ELN y otros grupos, incluidas las formaciones paramilitares formalmente desmanteladas, pero todavía activas, puedan llenar el vacío dejado por las Farc-Ep y así aprovechar el actual período de transición en que las Farc-Ep se desmovilizan y el Estado toma el control de grandes regiones anteriormente controladas por estas. También se sabe que no todas las unidades de las Farc-Ep se han desmovilizado y que existen unidades disidentes que todavía están activas en algunas partes del país (El Espectador, 2017).

No está claro qué papel desempeñan las actividades ilegales y las estructuras delictivas en la construcción o el menoscabo de una orden social en el posconflicto (Schultze-Kraft, 2017); mientras tanto, las vidas de los líderes de grupos sociales y medioambientales siguen siendo amenazadas. Por lo menos 389 agresiones (59 de las cuales eran asesinatos) contra líderes de derechos humanos fueron registradas en 2016 solamente (Human Rights Council, 2017), tristemente una de las tasas más altas el mundo; por último, muchas personas temen que el costo de la construcción de paz no pueda ser sostenido por el Estado colombiano, y que los fondos no alcancen 
las ambiciones del acuerdo de paz luego de la desaparición del interés y el apoyo financiero de las organizaciones internacionales.

En suma, muchas personas son muy escépticas y pesimistas, incluso resignadas, y tienden a considerar el acuerdo de paz como un acuerdo inútil, que en su esencia no es diferente a otras leyes y políticas fallidas, y que por lo tanto no suscita mucha esperanza. En un paralelo propuesto por algunas personas, el acuerdo de paz puede resultar similar a otros documentos supuestamente históricos, incluida la Constitución de 1991: después de mucha preparación y celebración, muchas personas no han experimentado los cambios significativos que esperaban y, más bien, han en gran parte visto sus esperanzas ser frustradas. En lo que tal vez es un mecanismo de autoprotección, tales personas no parecen estar preparadas para invertir emocionalmente, mucho menos políticamente, en este acuerdo de paz.

\section{Las raíces de la indiferencia}

La subestimación del conflicto armado en Sogamoso y la magnificación de la posibilidad de una paz fallida, sugieren que la indiferencia al acuerdo de paz no solo y no necesariamente está relacionada con un descontento general con la política, ni con la ignorancia de las apuestas del proceso de paz, aunque estos factores puedan jugar un papel. Esta justificación puede ser explicada principalmente por las fracturas que afectan profundamente a la sociedad colombiana.

\section{Los colombianos y el conflicto}

La indiferencia frente al proceso de paz tiene sus raíces en una fractura entre partes de la sociedad colombiana y el conflicto armado que ha plagado el país en formas diferentes, y por la acción de actores diferentes a lo largo de aun más de las cinco décadas de la insurgencia de las Farc-Ep. Se podría esperar que, dada la persistencia de guerras y conflictos en el país, sus causas y efectos hubieran sido atendidos en la identidad nacional y en el imaginario social y reivindicados por la mayoría de los colombianos. Sin embargo, a pesar de unas iniciativas verdaderamente sobresalientes (por ejemplo, CHNM, 2015), muchos colombianos ignoran las causas originales de las insurgencias de los campesinos, así como de las Farc-Ep. Como señaló Ospina (2013), el conflicto armado con las Farc-Ep no fue reconocido como una guerra por el gobierno colombiano durante mucho tiempo, reconocer la existencia de una guerra habría legitimado implícitamente al enemigo (Farc-Ep) y sus motivaciones, a su vez, habría arrojado dudas sobre la legitimidad de las élites que han mantenido el poder político y económico en Colombia desde la independencia.

Así, para muchos en Colombia, el conflicto con las Farc-Ep fue una lucha contra los grupos delincuentes y narcotraficantes, mientras que las causas originales de la movilización -la distribución desigual de la tierra y de los recursos, la falta de representación política en las poblaciones no criollas, la marginación de las minorías y de las poblaciones campesinas, el abandono de las zonas rurales por parte del Estado, la apropiación forzosa de los recursos naturales, el desconocimiento de la historia cultural, social y económica y del valor de los diversos territorios- se ignoran, a veces deliberadamente. De hecho, los que se oponen a las medidas de justicia transicionales en el acuerdo de paz suelen compartir esta interpretación del conflicto armado (Cárdenas, 2013; Ospina, 2013).

El no saber o, mejor, el no comprender plenamente las causas del conflicto, hace que muchos colombianos ignoren no solo las motivaciones originales de las Farc-Ep y otros grupos, sino también la diversidad de formas que la violencia tomó en el conflicto. El Centro Nacional de Memoria Histórica (CHNM, 2015) ha documentado ampliamente no solo los ataques, los asesinatos, las capturas de rehenes, el trastorno a la infraestructura, y otros acontecimientos infames muy visibles y más frecuentemente registrados, sino también los impactos inmateriales de la violencia que incluyen la angustia emocional, las humillaciones, el aislamiento físico y simbólico de las comunidades y los grupos sociales (indígenas, campesinos, minorías, mujeres y jóvenes), la injusticia y las amenazas a la dignidad humana, la falta crónica de instituciones democráticas y las restricciones a las libertades fundamentales (véase Feola et al., 2015 para una revisión de los efectos de los conflictos armados en el sector agrícola en Colombia).

Tal ignorancia hace más fácil poner distancia entre uno mismo y el conflicto, deliberadamente o no, y así reproducir la brecha de conocimiento sobre el conflicto. La ignorancia también obstaculiza la construcción de una memoria colectiva del conflicto en Sogamoso y otras ciudades y regiones que no se encontraron, o que se encontraron durante poco tiempo, en la primera línea del conflicto: los asesinatos; la llegada de desplazados forzosos de otras regiones; el papel de los centros urbanos como refugios donde los rebeldes de diferentes facciones descansarían o pasarían para trasladarse a las líneas de frente; las restricciones a la libertad de movimiento en la región y la angustia de vivir en la presencia casi constante de la violencia, sea localmente o sea difundida por los medios de comunicación desde otras partes del país.

Por lo tanto, esta brecha de conocimientos entre muchos colombianos sobre las razones históricas del conflicto armado en el país, justifica la explicación de la indiferencia que he resumido por el argumento "nunca ha habido conflicto en Sogamoso". Se sugiere que tan extendida como esta brecha es en el país, este hallazgo puede aplicarse a otras regiones colombianas que, como Sogamoso, no han estado en la primera línea del conflicto. Por otra parte, como propuesta por varios observadores (por ejemplo, Grajales, 2013; Richani, 2012), esta brecha 
de conocimiento ha sido en parte producida y perpetuada por las élites y aquellos actores sociales que más tuvieron que ganar con ella: aquellos cuyos intereses necesariamente chocan con cualquier serio intento a abordar la desigualdad, la desposesión, la exclusión social, cultural y política que dieron origen al conflicto armado en Colombia. Coherentemente, esta postura también minimiza la importancia de abordar algunas de las causas profundas del conflicto a través de la reforma rural y política incluida en el acuerdo de paz.

\section{Una sociedad dividida}

La sociedad colombiana está dividida rígidamente en múltiples líneas, de una manera que hace casi natural separar a "nosotros" de algunos "otros" (Herrera, 2016). Estas divisiones tienen raíces históricas y geográficas, como lo argumentan los historiadores que describen a Colombia como "un país fragmentado, una sociedad dividida" (Safford y Palacios, 2001) y una "nación a pesar de sí misma" (Bushnell, 1997). Colombia es un país "súpercomplejo" (Carrizosa, 2014), con una enorme diversidad cultural, medioambiental y étnica entre y dentro de las regiones, pero rara vez la diversidad ha sido vista como una riqueza, con la excepción quizá de algunos discursos más recientes alrededor de su biodiversidad (Carrizosa, 2014). En lugar de adoptar la diferencia para construir un proyecto nacional original, las élites colombianas han construido un Estado liberal solo formalmente moderno que no ha reconocido ni apreciado la diversidad (Ospina, 2013).

El Estado colombiano ha sido en su esencia un proyecto centralizador y distante de sus ciudadanos, los cuales no han sido adecuadamente incluidos en un imaginario nacional compartido (Herrera, 2016; Rueda, 2016). Los derechos de diversas poblaciones (indígenas, afrodescendientes, campesinas) que habitan el país han sido vistos como una barrera para un modelo particular de desarrollo económico, más que como su condición previa y esencial (Inter-American Commission on Human Rights, 2015), como es patente en la cultura de tolerancia para la represión violenta de la oposición, como la supresión del reciente paro agrario (Cruz, 2015) y la eliminación física de líderes y opositores progresistas, desde Jorge Eliécer Gaitán hasta los asesinatos de líderes de movimientos sociales (Ospina, 2013).

Así, las divisiones se han perpetuado y, si es posible, se han multiplicado a través de los siglos, también a través de la inacción de los poderes políticos. Esta inacción se ha caracterizado por la incapacidad de los sucesivos gobiernos colombianos para diseñar e implementar una reforma rural adecuada, algo que la mayoría de los gobiernos latinoamericanos han logrado hacer, aunque con resultados contradictorios (Oxfam, 2016; Rueda, 2016). Colombia tiene una de las concentraciones más altas de propiedad de la tierra en el mundo, lo que se explica, entre otros factores, por la distorsión de la toma de decisiones local por parte de las élites para beneficiarse (Grajales, 2011; 2016). La brecha entre centros urbanos relativamente ricos y zonas rurales pobres han estado creciendo durante décadas (Jaramillo, 2006; Rodríguez et al., 2016), al igual que la distancia entre los ricos y los pobres dentro de las ciudades.

Con el telón de fondo de estas múltiples fracturas sociales, no puede sorprender que muchas personas en Sogamoso y otras ciudades que fueron relativamente menos involucradas en el conflicto armado puedan percibir que el acuerdo de paz es algo en el que no tienen un interés significativo. Quizá es demasiado fácil y socialmente aceptable, en un país tan dividido, pensar que los acontecimientos potencialmente históricos, como el acuerdo de paz y el presente esfuerzo de construcción de paz, no nos interesan, sino que interesan a algún otro sujeto al otro lado de una o muchas fracturas sociales y geográficas. $\mathrm{O}$ pensar que el acuerdo de paz es simplemente una fachada noble detrás de la cual los poderosos pueden mantener el statu quo.

\section{Cuatro tesis contra la indiferencia}

En este artículo se han utilizado las perspectivas obtenidas de las conversaciones con los colombianos en Sogamoso para dar una explicación, tentativa e incompleta, a lo que parece ser indiferencia frente al acuerdo de paz en Colombia. Se ha resumido la explicación de la indiferencia en dos conjuntos de argumentos ("nunca ha habido conflicto en Sogamoso" y "nunca habrá paz en Colombia"), y se ha sugerido que estas ideas nos apuntan hacia la persistencia de profundas diferencias sociales, políticas, geográficas y culturales en Colombia. Una reflexión inmediata que se desprende de este material es que, bajo esta luz, sería demasiado simplista explicar apresuradamente la indiferencia y la separación solo con una desconfianza general en la política. Problemas más profundos y estructurales están en juego.

Un segundo punto de reflexión se refiere al esfuerzo de construcción de paz. Es un hecho que un número considerable de colombianos no se ha comprometido con el acuerdo de paz o la consolidación de la paz. ¿Acaso tal desinterés socavará el esfuerzo hacia la paz? Y si la indiferencia es una amenaza para el potencial cambio histórico, ¿cuáles son las razones por las que los colombianos deberían participar activamente en ese esfuerzo?

En síntesis, el argumento es el siguiente: a pesar de todas sus limitaciones, el acuerdo de paz y su proceso de construcción, representa una gran oportunidad para catalizar el cambio en Colombia, no solo para construir una paz estable y duradera, sino para hacer más incluyente el país y así también transformar algunas de las causas estructurales del conflicto; sin embargo, esto solo puede ocurrir si todos los colombianos, no solo la sociedad civil organizada, sino también grandes partes de la población, aprovechan esta oportunidad y participan activamente en 
un esfuerzo público y civil de construcción de la paz. La participación activa no solo significa apoyar al Gobierno y sus organismos y a las Farc-Ep, sino también apropiarse de esta paz colombiana y darle forma a través de actos políticos en espacios públicos, así como en lo cotidiano. El acuerdo de paz es una oportunidad, tal vez limitada e imperfecta, seguramente desafiante y difícil, no solo para superar décadas de conflicto, sino para conformar la identidad y las estructuras del país de una manera nueva, inclusiva, diversa, democrática, digna, y ambientalmente consciente.

Aquí, en forma de conclusión, se desarrolla este argumento a través de cuatro tesis contra la indiferencia.

\section{Primera tesis. Las limitaciones del acuerdo de paz no eliminan su potencial de cambio}

El acuerdo de paz es imperfecto y la construcción de paz es seguramente un esfuerzo enormemente desafiante que puede inducir a muchos colombianos a pensar de que nunca habrá paz en Colombia; sin embargo, es difícil negar que el acuerdo de paz contiene, al menos potencialmente, las semillas de muchas reformas estructurales y radicales que son necesarias y que abordarían de manera positiva, aunque tal vez incompleta, algunas de las cuestiones estructurales que han ido caracterizando a Colombia por largo tiempo, las cuales originalmente motivaron a las Farc-Ep y otros grupos a la insurgencia. Por ejemplo, la Reforma Rural Integral aborda algunos de los problemas que han afectado históricamente la agricultura y las zonas rurales, como el acceso a la tierra, la formalización de los títulos de propiedad, el acceso al crédito, el apoyo técnico y la capacitación, la infraestructura, el reconocimiento social y cultural de los campesinos, de la agricultura y del desarrollo sostenible.

La Reforma de Participación Política promete abrir el sistema democrático y ofrecer una representación de las minorías en el país, mientras que la Comisión de la Verdad puede producir la evidencia necesaria para construir no solo una memoria compartida del conflicto armado, sino un clima de reconciliación; además, el proceso de paz ya ha tenido efectos en la vida política del país, ha renovado el debate nacional sobre los grandes desafíos que enfrenta Colombia, ha reforzado el papel y reafirmado el valor de un debate político pacífico y respetuoso entre antiguos enemigos, ha incitado a pensar sobre la justicia social de manera territorial y ha permitido recuperar la memoria de la guerra (Rueda, 2016).

No hay un acuerdo completo sobre los principios en que se basan las reformas incluidas en el acuerdo de paz (Carmona, 2015), además, algunos elementos de estas reformas no son totalmente nuevos, ya se incluyeron en textos legislativos anteriores que no se han implementado o aplicado con éxito. Sin embargo, el acuerdo de paz reitera la necesidad de promulgar estas reformas, y cuando se traduzca en legislación proporcionará una base legal para su implementación. Esto no es insignificante, como ilustra el caso de la constitución ecuatoriana de 2008.

Ecuador dio la bienvenida a una nueva constitución en el 2008. Esta constitución fue celebrada nacional e internacionalmente como la formalización innovadora de un cambio hacia una idea posneoliberal del desarrollo, se esperaba que la nueva constitución apoyara el cambio social, cultural, institucional y económico necesario para realizar esa visión alternativa. La constitución formalizó un modelo de estado plurinacional e intercultural que tiene en cuenta a los ciudadanos diferenciados y a las reivindicaciones históricas de las primeras naciones (Radcliffe, 2012). La constitución de 2008 estableció los derechos sociales como un derecho constitucional, reconoció más de 100 derechos para los ciudadanos y reformó el sistema de justicia con el objetivo de garantizar que todos los nuevos derechos se protejan a través de los tribunales. En particular, la Constitución de Ecuador de 2008 es también innovadora en términos de su reconocimiento de los derechos de la naturaleza.

Sin embargo, con mucha frustración dentro y fuera del país, el Gobierno ecuatoriano ha legislado y actuado repetidamente contra los principios constitucionales, por ejemplo, en los temas de extracción (petróleo) y manejo (agua) de recursos naturales (Burchardt y Dietz, 2014). Sin embargo, la nueva constitución no puede descartarse como un fracaso, la aprobación misma de una carta tan innovadora y ambiciosa ha cambiado el discurso y ha creado espacios políticos para repensar el desarrollo no solo en Ecuador sino internacionalmente. El poder simbólico de la constitución ha inspirado a grupos indígenas, críticos académicos y políticos, y movimientos sociales en Ecuador e internacionalmente a desafiar modelos neoliberales de desarrollo y explorar visiones y prácticas alternativas como Buen Vivir y sumak kawsay (Gudynas, 2011). Además, la constitución ha proporcionado una base legal para resistir la explotación y la apropiación violenta de los recursos naturales y la interrupción de los sustentos sostenibles por el neoliberalismo, como en el caso de las luchas por defender territorios sagrados contra el extractivismo (Harris y Roa-García, 2013). Si bien el cambio sociolegal formalizado en la nueva constitución no es ciertamente suficiente para derrocar los modelos de desarrollo neoliberales, ofrece una herramienta legal para resistir la influencia de las instituciones financieras internacionales, las corporaciones transnacionales y las prevaricaciones del gobierno nacional cuando no actúa de acuerdo con la Constitución (Harris y Roa-García, 2013; Radcliffe, 2012).

Así, el caso ecuatoriano muestra cómo las políticas históricas, por imperfectas y sujetas a apropiación de intereses dominantes, pueden ayudar a apoyar la lucha por la igualdad, la justicia social, la democracia, la participación y la sostenibilidad. Estos documentos no solo inspiran, sino que, lo que es más importante, proporcionan la base 
legal para desafiar el desvío de los principios básicos. El caso de la constitución ecuatoriana también muestra que una condición esencial para realizar el potencial del cambio que está latente en estos documentos es su apropiación activa por parte de la sociedad civil (Becker, 2011); exactamente porque estos documentos y su constelación política son imperfectos, es esencial que la sociedad civil no espere que el proceso institucional cuide de sí mismo, sino que se apropie del documento y defienda activamente sus principios contra cualquier amenaza de dilución, desvió o interrupción.

\section{Segunda tesis. La presión de la sociedad civil y del público puede hacer que los que tienen el poder directo por su implementación rindan cuentas de la realización del acuerdo de paz}

Aun reconociendo que el acuerdo de paz está rodeado por retórica, es difícil ignorar que tanto el Gobierno colombiano como las Farc-Ep han invertido un capital simbólico y financiero muy importante al comprometerse con el acuerdo de paz, ellos tienen la responsabilidad principal y directa de la aplicación del acuerdo y son aquellos cuya acción será examinada más de cerca y que serán juzgados por los resultados del proceso de paz.

Sin embargo, existe el riesgo concreto de que el acuerdo de paz no se lleve a cabo integralmente debido, entre otras cosas, a la decandencia de la motivación de las partes involucradas, la disminución de los recursos financieros, la falta de voluntad política o capacidad técnica; sin embargo, un factor tiene eficacia probada contra todas esas causas posibles de falla: la presión pública. El público colombiano, la sociedad civil, y los medios de comunicación de masas tienen un papel que desempeñar para presionar a todas las partes implicadas para que realicen plenamente el acuerdo, priorizando la paz en la agenda política y dedicándole los recursos financieros, técnicos, y políticos necesarios. La sociedad civil y los medios de comunicación pueden responsabilizar a los que tienen poder directo por la aplicación del acuerdo, pueden exigir que se implemente el acuerdo de paz y que esto se haga de forma transparente.

Resulta inspirador ver que algunas iniciativas ya se han puesto en marcha exactamente con el propósito de monitorear el progreso de la implementación del acuerdo de paz. Por ejemplo, la Fundación Paz y Reconciliación desarrolló un semáforo del posconflicto para hacer seguimiento al progreso en la implementación del acuerdo de paz; esta es una herramienta fácil de usar por los ciudadanos y los medios de comunicación, la cual complementa los más técnicos informes de investigación de la Fundación. Iniciativas como estas ganan fuerza cuando son apoyadas, en línea y fuera de línea, por otras organizaciones y por el público.

Claramente, lo contrario de la presión pública -la indiferencia- no motivará al Gobierno colombiano ya las
Farc-Ep, ni a las organizaciones internacionales a cumplir con su compromiso. La indiferencia al acuerdo de paz no elimina su existencia, sino que silencia a los ciudadanos, los cuales, les guste o no, serán afectados por las consecuencias de una implementación exitosa o fallida del acuerdo de paz. No es a través de la indiferencia, sino a través de la participación civil y el apoyo mutuo entre aquellos que creen que la paz es mejor que la guerra, que un gran frente de presión pública puede mantenerse y las posibilidades de realizar la promesa de paz se pueden aumentar.

\section{Tercera tesis. La sociedad civil y el público pueden apropiarse del proceso de paz e influir en la construcción de paz}

Responsabilizar directamente a los actores que tienen el poder directo para la aplicación del acuerdo de paz es solo una de las funciones de la sociedad civil organizada y del público; de hecho, ellos pueden ir más allá: pueden influir en la dirección, la velocidad, y la realización concreta del proceso de construcción de paz. La sociedad civil puede convertirlo en su propio proyecto si se apropia de la paz en lugar de observar pasivamente su progreso, contradictorio y tembloroso, o la falta de ello, desde afuera. $\mathrm{Si}$ es cierto que los intereses dominantes tienen la capacidad de desviar el proceso de paz a nivel institucional, la sociedad civil puede responder a través de un contra desvío en la esfera pública. Una de las lecciones que se pueden sacar de la experiencia de las iniciativas de paz desde la base en Colombia en las últimas décadas es que las interconexiones entre las organizaciones de la sociedad civil pueden incrementar las probabilidades de éxito y fortalecer la conciencia colectiva y la reflexión sobre la paz (Hernández, 2009). Por ejemplo, un proceso de paz civil puede apoyar la implementación de una paz maximalista, en lugar de una paz minimalista (Ramos, 2016; Rettberg, 2003): una paz que genera las condiciones para el desarrollo económico, político social y lo hace en respeto a la diversidad, los derechos humanos y el medio ambiente.

Es importante hacer hincapié a las muchas experiencias concretas en Colombia, a menudo dirigidas por mujeres y grupos indígenas, sobre las cuales se puede aprender y construir (por ejemplo, CIASE y Consiliation Resources, 2017; Colectivo de Pensamiento y Acción Mujeres, Paz y Seguridad, 2017; Hernández, 2009; Mera, 2013; Sánchez y Rodríguez, 2015). Es en estas experiencias concretas que la paz ya se está construyendo en Colombia no solo por el Estado, sino desde la base de muchas iniciativas de resistencia de la sociedad civil y actos cotidianos para "la convivencia pacífica, la gestión y la resolución no violenta de conflictos" (Hernández, 2009, p. 132). "La construcción de paz no solo encuentra su origen en el Estado y los procesos de negociación de paz. En este país la paz también se construye a niveles de base social y en una dimensión de abajo hacia arriba" (Hernández, 2009, p. 132; Moriconi, 
2011). Los actos políticos (protestas, huelgas) pueden ser importantes, pero la historia nos ha demostrado la importancia igual, si no mayor, de los actos cotidianos aparentemente menores de concienciación y reconocimiento en la construcción de una cultura de inclusión, paz y democracia a pesar de adversos contextos institucionales y políticos (Snyder, 2017). Los actos cotidianos de vida pacífica pueden concretizar el imaginario de la paz y multiplicar su discurso y práctica (Parra, 2013): la paz no es solo una construcción legal, sino "una construcción subjetiva y social de la gente que vive y experimenta cotidianamente" (Herrera, 2016, p. 53). Ellos representan una política como polis (cívica), en lugar de una política como policy (técnica; Moriconi, 2011).

Es importante destacar que una apropiación de la construcción de paz en Colombia también es la apropiación de una parte de la responsabilidad del resultado de este proceso; por lo tanto, pide un alejamiento de la indiferencia y una reclamación y ejercicio pleno de ciudadanía (Parrado, 2013), es decir, conciencia y reclamación del papel de los ciudadanos no como objetos pasivos, sino como agentes activos de cambio social, lo cual se ilustra a través de las muchas iniciativas ya existentes de construcción de paz desde abajo en el país. Pero esto no puede limitarse a algunas iniciativas, aunque sobresalientes y esperanzadoras, y no puede dejar de lado al público colombiano - los ciudadanos colombianos- ya que esto perpetuaría la fractura entre unas élites y el resto de la sociedad (Urrego y Betancour, 2016). En palabras de William Ospina:

Tenemos que olvidar el viejo error de pensar que unos cuantos elegidos se encargarán de transformar el país y salvarnos de la adversidad. Colombia necesita un pueblo entero comprometido en su transformación. Necesita creer profundamente que el poder no está en una silla lejos del mundo, que el poder está en cada lugar (Ospina, 2013, p. 235).

\section{Cuarta tesis. La construcción de paz significa la oportunidad de construir no sólo la paz, sino una Colombia más diversa, inclusiva, democrática, digna y ambientalmente consciente}

El problema más grave de la indiferencia es que condena la invisibilidad. Un público que es indiferente efectivamente declina los derechos y deberes de la plena ciudadanía, y se vuelve invisible para los que están en el poder; así, un público invisible es una contraparte perfecta para cualquier élite que quisiera perseguir su propio interés en vez del interés público, esto es exactamente lo que muchas de las personas con las que se discutió en Sogamoso temían. En un país tan dividido como Colombia, donde las élites históricamente han perpetuado y aprovechado divisiones étnicas, geográficas y sociales, y donde las divisiones han causado y justificado la violencia, el despojo, la explotación, la exclusión y la desigualdad, la indiferencia al acuerdo de paz equivaldría, en otras palabras, a negar que existe la posibilidad de desafiar y recomponer las fracturas de este país dividido, y al mismo tiempo la posibilidad de resolver algunas de las causas estructurales más profundas del conflicto armado.

La cita de Ospina que abre este artículo no es solo un llamado a un compromiso civil, sino una exhortación a pensar en el acuerdo de paz como una oportunidad no solamente para construir paz. Se trata de una invitación a utilizar la construcción de paz para hacer frente a problemas sociales, políticos, medioambientales y de desarrollo de larga data que no se han podido relacionar en el discurso común del conflicto pero que han afectado al país y limitado su consolidación democrática, la inclusión social y la aparición de formas endógenas de desarrollo. Esto puede parecer un objetivo aún más ambicioso y, por lo tanto, lleno de ambigüedad y peligros. ¿No hay aún más razones para ser pesimista y escéptico frente a la transformación de Colombia, no solamente para construir paz?

Y, sin embargo, ¿qué pasaría si fuera exactamente la paz la que diera la oportunidad de unir fuerzas para el objetivo más alto? ¿Y si el acuerdo de paz creara el impulso de que hasta ahora han carecido las muchas iniciativas para la paz y la transformación social? ¿Qué pasaría si la paz fuera el terreno común en el que convergieran y se unieran diferentes grupos e intereses? ¿Qué pasaría si las propias élites hubieran ofrecido a la población, quizá involuntariamente, un discurso que conecte instancias sociales, ambientales, culturales y políticas que hasta ahora se han mantenido separadas unas de otras? ¿Y si este fuera un momento en que el sistema de intereses dominantes fuera más vulnerable a una transformación?

Muchos movimientos sociales en Colombia ya están avanzando en sus luchas, experimentando y proponiendo transformaciones democráticas locales, cruzando intereses particulares como lo ilustra, por ejemplo, el Congreso de los Pueblos: las fracturas pueden ser superadas, las identidades colectivas pueden ser creadas, y la diversidad puede ser una fuente de dignidad; no tiene que ser fuente de violencia. Estos movimientos han sentado las bases para una "política de civilización" (Moriconi, 2011), que es una política que no busca aumentar el bienestar, sino el bien-vivir, y apoyar modelos pacíficos y socialmente integradores. A nivel internacional, varios observadores han exigido que se establezcan similares interconexiones y colaboraciones entre movimientos medioambientales, sociales, feministas e indígenas, entre otros (por ejemplo, Klein, 2015; 2017). Los ejemplos actuales de estos movimientos de movimientos son alentadores (Klein, 2017), pero a menudo están limitados por la falta de apoyo y participación activa del público. Lo que puede faltar o debilitarse en Colombia es la capacidad de los ciudadanos 
para superar las diferencias y la alteridad para construir una identidad colectiva (Herrera, 2016).

Se busca sugerir que el acuerdo de paz es un catalizador adicional de iniciativas, energías y visiones. La paz puede proporcionar un imaginario interseccional, un discurso y una práctica cotidiana en torno a la cual no solo los movimientos sociales, sino todos los colombianos pueden reunirse en la visión y la realización de un país pacífico y nuevo. La paz (no una paz mínima entendida como ausencia de conflicto armado con un grupo insurgente, sino una paz multidimensional y maximalista) ofrece la oportunidad histórica de recomponer las fracturas y de así transformar la sociedad colombiana.

Las conversaciones en Sogamoso han demostrado que incluso los colombianos que han sido sustancialmente indiferentes al acuerdo de paz en realidad están de acuerdo con él, al menos en parte o en principio. Este nivel latente de acuerdo, sin embargo, permanecerá invisible mientras el público se desvincule del proceso de paz. La indiferencia no es una posición neutral de equidistancia entre un sí y un no. La indiferencia condena a la invisibilidad y, por lo tanto, presta apoyo implícito a los que se oponen a la paz. Como tal, la indiferencia corre el riesgo de convertir el fracaso del proceso de paz en una profecía auto cumplida. Para evitar esto, se espera que el pueblo colombiano responda a la llamada de la historia comprometiéndose a construir la paz en el país.

\section{Referencias}

Alto Comisionado para la Paz (2016). Acuerdo final para la terminación del conflicto y la construcción de una paz estable y duradera. Bogotá. Recuperado de http://www.altocomisionadoparalapaz. gov.co/procesos-y-conversaciones/Documentos\%20compartidos/24-11-2016NuevoAcuerdoFinal.pdf

Becker, M. (2011). Correa, indigenous movements, and the writing of a new constitution in Ecuador. Latin American Perspectives, 38(1), 47-62. https://doi.org/10.1177/0094582X10384209

Burchardt, H., y Dietz, K. (2014). (Neo-) extractivism-a new challenge for development theory from Latin America. Third World Quarterly, 35(3), pp. 468-486. https://doi.org/10.1080/01436597.2014 .893488

Bushnell, D., (1997). Colombia, una nación a pesar de sí misma: de los tiempos precolombinos a nuestros días. Barcelona: Planeta.

Cárdenas, J. (2013). Opinión pública y proceso de paz: actitudes e imaginarios de los bogotanos frente al proceso de paz de La Habana entre el gobierno colombiano y la guerrilla de las FARC. Ciudad Paz-ando, 6(1), pp. 41-58. https://doi.org/10.14483/udistrital.jour. cpaz.2013.1.a03

Carrizosa, J. (2014). Colombia Compleja. Bogotá: Jardín Botánico José Celestino Mutis, and Instituto de Investigación de Recursos Biológicos Alexander von Humboldt.

Centro Nacional de Memoria Historica (CNMH). (2015). ¡Basta Ya! Colombia: memorias de guerra y dignidad. Bogotá: Centro Nacional de Memoria Histórica.
Colectivo de Pensamiento y Acción Mujeres, Pazy Seguridad (2017). Pacto ético por un país en paz. Recuperado de http://pactoetico.org/

Cruz, E. (2015). El derecho a la protesta social en Colombia. Piensamento Juridico, 42, 47-69.

Diaz, F. (2017) Colombians are fed up with corruption, and everyone seems to be under investigation. The Conversation. Recuperado de https://theconversation.com/colombians-are-fed-up-with-corruption-and-everyone-seems-to-be-under-investigation-75173

El Espectador. (Mayo 6 de 2017). La disidencia de las Farc que le dijo no a la paz. Recuperado de https://colombia2020.elespectador. $\mathrm{com} /$ pais/la-disidencia-de-las-farc-que-le-dijo-no-la-paz

El País. (Octubre 3 de 2016). Así votaron los colombianos. Recuperado de https://elpais.com/elpais/2016/10/03/media/1475486923_970895.html

Faguet, J, Sanches, F. y Villaveces, M. (2016). The paradox of land reform, inequality and local development in Colombia. London: The London School of Economics and Political Science.

Feola, G. (2017). Adaptive Institutions? Peasant Institutions and Natural Models facing climatic and economic changes in the Colombian Andes. Journal of Rural Studies, 49, 117-127. https://doi. org/10.1016/j.jrurstud.2016.10.007

Feola, G., Agudelo, L. y Bamón, B. (2015). Colombian agriculture under multiple exposures: a review and research agenda. Climate and Development, 7(3), 278-292. https://doi.org/10.1080/1756552 9.2014 .934776

González, M. (2017). La "posverdad" en el plebiscito por la paz en Colombia. Nueva Sociedad, 269, 114-126.

Grajales, J. (2011). The rifle and the title: paramilitary violence, land grab and land control in Colombia. The Journal of Peasant Studies 38, 771-792. https://doi.org/10.1080/03066150.2011.607701

Grajales, J. (2013). State Involvement, Land Grabbing and Counter Insurgency in Colombia. Development and Change, 44(2), 211-232. https://doi.org/10.1111/dech.12019

Gudynas, E. (2011). Buen Vivir: Today's tomorrow. Development, 54, 441-447. https://doi.org/10.1057/dev.2011.86

Harris, L., Roa-García, M. (2013). Recent waves of water governance: Constitutional reform and resistance to neoliberalization in Latin America (1990-2012). Geoforum, 50, 20-30. https://doi.org/10.1016/j.geoforum.2013.07.009

Herbolzheimer, K. (2016). Innovations in the Colombian peace process. Oslo: The Norwegian Peacebuilding Resource Centre.

Hernández, E. (2009). Resistencias para la paz en Colombia. Experiencias indígenas, afrodescendientes y campesinas. Revista de Paz y Conflictos, 2, 117-135.

Hernández, E. (2015). Empoderamiento Pacifista del actual proceso de paz en Colombia: 2012-2015. Revista de Paz y Conflictos, 8(2), 179-202.

Herrera, D. (2016). La paz subjetiva y plural. En: Gentili, P., Alvarado, S. V., Rueda Barrera, E. A., (Editors). Paz en Colombia: Perspectivas, desafios, opciones. Buenos Aires: CLACSO. 
Human Rights Council (2017). Annual Report of the United Nations High Commissioner for Human Rights on the situation of human rights in Colombia. Recuperado de http://www.ohchr.org/EN/ HRBodies/HRC/RegularSessions/Session34/Pages/ListReports.aspx

Inter-American Commission on Human Rights (2015). Indigenous Peoples, Afro-Descendent Communities, and Natural Resources: Human Rights Protection in the Context of Extraction, Exploitation, and Development Activities. Washington: OEA.

Jaramillo, P. (2006). Pobreza rural en Colombia. Revista Colombiana de Sociología, 27, 47-62.

Klein, N. (2015). This changes everything: Capitalism vs. the climate. London: Simon and Schuster.

Klein, N. (2017). No Is Not Enough. London: Allen Lane.

Mera, A. (2013). Experimentando con la sensibilidad al conflict en un laboratorio de construcción de paz. Ciudad Paz-ando, 6(1), 59-78. https://doi.org/10.14483/udistrital.jour.cpaz.2013.1.a04

Moriconi, M. (2011) Desmitificar la violencia: crítica al discurso (técnico) de la seguridad ciudadana. Revista Mexicana de Sociología, 73(4), 617-643.

Nasi,C.andRettberg,A.(2016).Colombia'sFarewelltoCivilWar:Reaching Closure in a Divided Society. Recuperado de https://poseidon01. ssrn.com/delivery.php?ID=799026031026005029084002084075 09611110903602108209005209311501907002600909711710002 5049022043006054025039123112004107068065003005043060 00802100907708808808502409110306903808310411108411212 107407209811511710711202506712409611409909910906412208 612 3073024\&EXT $=$ pdf

Ospina, W. (2013). Pá que se acabe esta vaina. Barcelona: Planeta.

Oxfam (2016). Desterrados: Tierra, Poder y Desigualidad en Américal Latina. Oxfam. Recuperado de https://www.oxfam.org/es/informes/desterrados-tierra-poder-y-desigualdad-en-america-latina

Parra, M. (2013). Hablando sobre paz, ¿actuando por la paz?. Ciudad Paz-ando, 6(1), 177-182. https://doi.org/10.14483/udistrital.jour. cpaz.2013.1.a10

Radcliffe, S.(2012). Development for a postneoliberal era? Sumak kawsay, living well and the limits to decolonisation in Ecuador. Geoforum 43, 240-249. https://doi.org/10.1016/j.geoforum.2011.09.003

Ramos, E. (2016). El proceso de construcción de paz colombiano más allá de la negociación: una propuesta desde la Paz Transformadora y Participativa. El Ágora U.S.B., 16(2), 513-532.

Reconciliation Resources (2017). Indigenous women and Colombia's peace process: pathways to participation. London: Conciliation.
Rettberg, A. (2003) Diseñar el futuro: una revisión de los dilemas de la construcción de paz para el postconflicto. Revista de Estudios Sociales, 15, 15-28.

Richani, N. (2012). The agrarian rentier political economy. Land Concentration and Food Insecurity in Colombia. Latin American Research Review, 47, 51-78. https://doi.org/10.1353/lar.2012.0025

Rodríguez, J., Pardo, R., Acosta, O. y Uribe, L. (2016). Bienes y servicios públicos sociales en la zona rural de Colombia: brechas y politicas públicas. Bogotá: CEPAL.

Rodríguez, T. (2016). Geografía del terrorismo en Colombia: Una visión retrospectiva. Revista de Paz y Conflictos, 9(2),179-198.

Rueda, E. (2016). Paz en Colombia: Decolonización y cultura política. En: Gentili, P., Alvarado, S. y Rueda, E. (Eds.). Paz en Colombia. Perspectivas, desafios, opciones. Buenos Aires: CLACSO.

Safford, F. y Palacios, M., (2001). Colombia: Fragmented Land, Divided Society. Oxford: Oxford University Press.

Salas, L. (2015). Lógicas territoriales y relaciones de poder en el espacio de los actores armados: un aporte desde la geografía política al estudio de la violencia y el conflicto armado en Colombia, 19902012. Cuadernos de Geografia-Revista Colombiana de Geografia, 24(1), 157-172. https://doi.org/10.15446/rcdg.v24n1.47777

Schultze-Kraft, M. (2017) Making peace in seas of crime: crimilegal order and armed conflict termination in Colombia. Crime, Law and Social Change. Recuperado de https://link.springer.com/article/10.1007/s10611-017-9759-2

Siegfried, K. (2016). What Colombia's peace process can teach the world. IRIN News. Recuperado de https://www.irinnews.org/ analysis/2016/07/20/what-colombia\%E2\%80\%99s-peace-process-can-teach-world

Snyder, T. (2017). On tyranny: Twenty lessons from the twentieth century. New York: Tim Duggan Books.

The Guardian (Diciembre 1 de 2016). Colombia's government formally ratifies revised Farc peace deal. Recuperado de https:// www.theguardian.com/world/2016/dec/01/colombias-government-formally-ratifies-revised-farc-peace-deal

The Guardian, (Noviembre 24 de 2016). Colombia signs historic peace deal with Farc. Recuperado de https://www.theguardian.com/ world/2016/nov/24/colombia-signs-historic-peace-deal-with-farc-rebels

Urrego, G. y Betancour, L. (2016). Participación ciudadana: acción política y pluralidad en el acuerdo de paz en Colombia. Aproximaciones a referentes internacionales. Ciudad Paz-ando, 9(1), 117-135. 